\title{
Stability of toroidal magnetic fields in the solar tachocline
}

\author{
R. Arlt, A. Sule, and G. Rüdiger
}

\author{
Astrophysikalisches Institut Potsdam, An der Sternwarte 16, Germany \\ e-mail: rarlt@aip.de
}

Received 13 March 2006 / Accepted 4 September 2006

ABSTRACT

\begin{abstract}
The stability of toroidal magnetic fields in the radiative interior of the Sun is studied in a three-dimensional environment. The underlying differential rotation in the tachocline depends on both latitude and radius. We found stability limits for toroidal fields of only $100 \mathrm{G}$ even in the simplified, incompressible, but stably stratified case. Nonaxisymmetric modes are linearly unstable with a weak influence on the amplitude of the underlying differential rotation. We conclude that the tachocline is not a suitable location for the storage of magnetic fields as strong as $100 \mathrm{kG}$. Since the results are based on an extrapolation of the numerical computations to lower solar diffusivities, high-resolution calculations at higher magnetic Reynolds numbers are required.
\end{abstract}

Key words. Sun: magnetic fields - instabilities - Sun: rotation - magnetic fields - magnetohydrodynamics (MHD)

\section{Motivation}

The solar tachocline is the transition layer between the convection zone and the radiative solar core. The convection zone rotates differentially where the angular velocity $\Omega$ mainly depends on the latitude. The solar core rotates rigidly to a fairly high degree. The tachocline region is a place where a strong radial dependence of the angular velocity is also observed, so that it matches the uniform $\Omega$ in the core. The thickness of the tachocline is roughly $5 \%$ of the solar radius. The internal rotation profile outlined with these main facts has been confirmed by recent helioseismology.

A plausible formation mechanism for the tachocline is magnetic, as was proposed by Rüdiger \& Kitchatinov (1997) and Gough \& McIntyre (1998). The magnetic coupling of a magnetic field in the solar core with the differential rotation of the convection zone leads to a thin transition layer, thereby causing toroidal magnetic fields of about 200 Gauss. If the tachocline was the place where most of the magnetic flux is stored, which gives rise to solar activity, the field strength must be strong enough to give rise to visible emergence at the surface, as noticed by the appearance of sunspots. Field strengths of at least $10^{5}$ Gauss have been proposed as necessary for the emergence of flux at the surface on latitudes of $30^{\circ}$ or lower (Choudhuri \& Gilman 1987). This also explains the tilt angle of bipolar sunspot groups (D'Silva \& Choudhuri 1993).

The stability of magnetic fields in terms of flux tubes has been investigated thoroughly by Ferriz-Mas \& Schüssler (1995). The stability limits of toroidal fields were all beyond $10^{4}$ Gauss. The problem was tackled with ideal MHD on magnetic flux tubes, which we can understand as magnetic-feld belts that are only extended in the azimuthal direction, but not in the latitudinal and radial directions. We are instead approaching the stability issue with non-ideal MHD and with latitudinally and radially extended field belts. The influence of the thickness of the field belts and of the magnetic Prandtl number, which is the ratio of viscosity over magnetic diffusivity, can be studied.

The stability of purely toroidal magnetic fields against adiabatic perturbations were investigated analytically by
Tayler (1973). It was found that a large class of the configurations of toroidal fields is unstable in the linear treatment. Non-axisymmetric modes were more unstable than axisymmetric modes. The 2D study by Gilman \& Fox (1997) found a joint instability of differential rotation and magnetic fields with heavy destabilization of any amplitude of differential rotation in the presence of toroidal magnetic fields. For storing toroidal fields of $10^{5}$ Gauss in the tachocline over periods of time of the order of $2 \mathrm{yr}$, magnetic diffusivities of $3 \times 10^{10} \mathrm{~cm}^{2} / \mathrm{s}$ are necessary according to a more recent 2D approach by Dikpati et al. (2004).

We tackle the problem with a 3D setup assuming that the diffusivity is not much larger than the microscopic value. We ask for the maximum possible stable toroidal fields being subject to non-axisymmetric perturbations. The field belts are thin but of finite extent, and the full radial and latitudinal dependences of the magnetic-field structure and rotation profile are included. The numerical results are extrapolated to real-Sun values of magnetic fields in physical units. We have simplified the model to the incompressible MHD equations and can apply either adiabatic or stable stratification. If instability is found for fields significantly smaller than $10^{5} \mathrm{G}$ in such a simplified model, there will be even less hope for stable fields if compressibility is added, allowing for Parker instability and the like. The computations may also be interesting for the MHD laboratory experiments that are currently being designed for the study of astrophysical magnetic phenomena (Rüdiger et al. 2006).

The following section will deal with the numerical requisites and the capabilities of the numerical treatment. Section 3 contains results for the stability of toroidal fields in a rigidly rotating spherical shell. Section 4 deals with the influence of the thickness of the field belts. The computations are extended to differentially rotating spherical shells in Sect. 5 , followed by the discussion in Sect. 6.

\section{Numerical method}

Since this study aims at the stability of a given background state against small non-axisymmetric perturbations, the problem is 
formulated by linearized equations. We employ the incompressible, viscous Navier-Stokes equation for the velocity, as well as the diffusive induction equation for the magnetic field. The Navier-Stokes equation contains a Lorentz force, but neglects the effect of buoyancy.

The computational domain is a spherical shell where $r, \theta$, and $\phi$ denote the radius, colatitude, and azimuth, respectively. After linearization, we can separate the axisymmetric background rotation $\boldsymbol{U}=(0,0, r \sin \theta \Omega)$ from the nonaxisymmetric flow $\boldsymbol{u}$, with $\Omega$ being a normalised angular velocity. An imposed axisymmetric toroidal magnetic field $\boldsymbol{B}=\left(0,0, B_{\phi}\right)$ is also separated from the perturbations $\boldsymbol{b}$. The lower-case quantities are evolved by numerical computations. The upper-case quantities may depend on $r$ and $\theta$, but not on $\phi$. The linear, normalised incompressible MHD equations are

$$
\begin{aligned}
\frac{\partial \boldsymbol{u}}{\partial t}= & \operatorname{Rm}[\boldsymbol{u} \times \nabla \times \boldsymbol{U}+\boldsymbol{U} \times \nabla \times \boldsymbol{u}-\nabla(\boldsymbol{u} \cdot \boldsymbol{U})] \\
& +S[(\nabla \times \boldsymbol{b}) \times \boldsymbol{B}+(\nabla \times \boldsymbol{B}) \times \boldsymbol{b}] \\
& -\nabla p+\operatorname{Pm} \triangle \boldsymbol{u}, \\
\frac{\partial \boldsymbol{b}}{\partial t}= & \nabla \times(\operatorname{Rm} \boldsymbol{U} \times \boldsymbol{b}+S \boldsymbol{u} \times \boldsymbol{B})-\Delta \boldsymbol{b},
\end{aligned}
$$

and the relations $\nabla \cdot \boldsymbol{u}=0$ and $\nabla \cdot \boldsymbol{b}=0$ hold. The constant density $\rho$ and the magnetic permeability $\mu$ do not appear in this normalisation.

The equations are evolved with the spectral spherical code by Hollerbach (2000). The actual integrated quantities are the toroidal and poloidal potentials of $\boldsymbol{u}$ and $\boldsymbol{b}$. These are defined by $\boldsymbol{u}=\nabla \times(e \hat{\boldsymbol{r}})+\nabla \times \nabla \times(f \hat{\boldsymbol{r}})$ and $\boldsymbol{b}=\nabla \times(g \hat{\boldsymbol{r}})+\nabla \times \nabla \times(h \hat{\boldsymbol{r}})$, where $\hat{\boldsymbol{r}}$ is the radial unit vector. This construction ensures divergencefree velocities and magnetic fields at any time of the simulations. It also permits the easy cancellation of poloidal flows, so that purely horizontal flows can be studied for stably stratified media as well.

We look for exponentially decaying or growing solutions in order to find the critical magnetic background field strength $B_{\phi}$ of the marginal case. The actual integration of (1) employs the radial components of the curl and the curl-curl of the equation, thereby eliminating the gradient terms. The viscous part of the equation of motion is evolved implicitly, while the other parts are integrated explicitly with the advection term and forces being computed in real space. We kept this splitting even in our linearized problem, since a fully implicit scheme would have required a substantial modification of the code. The $\Delta \boldsymbol{u}$ is thus treated implicitly, the other rhs terms (four remaining after curling) are computed in real space and are used for a second-order Runge-Kutta integration.

The normalisation of the equation with the diffusion time $\tau_{\eta}=R^{2} / \eta$, the length scale $R$, and the magnetic diffusivity $\eta$ leads to the magnetic Reynolds number

$\mathrm{Rm}=\frac{R^{2} \Omega_{\mathrm{eq}}}{\eta}$

as a free parameter that is essentially a variation of the viscosity $\eta$ since the solar radius $R$ and the equatorial angular velocity $\Omega_{\mathrm{eq}}$ are sufficiently well known. Velocities are thus measured in units of $\eta / R$ and magnetic fields in units of $\sqrt{\mu \rho} \eta / R$. The normalisation replaces the viscosity of (1) by the magnetic Prandtl number $\mathrm{Pm}=v / \eta$.

In these units, the computational domain covers the radial extent from $r_{\mathrm{i}}=0.5$ to $r_{\mathrm{o}}=0.7$ and both hemispheres in latitude, $\theta=0$ to $\pi$. The outer radius is thought of as the radius at

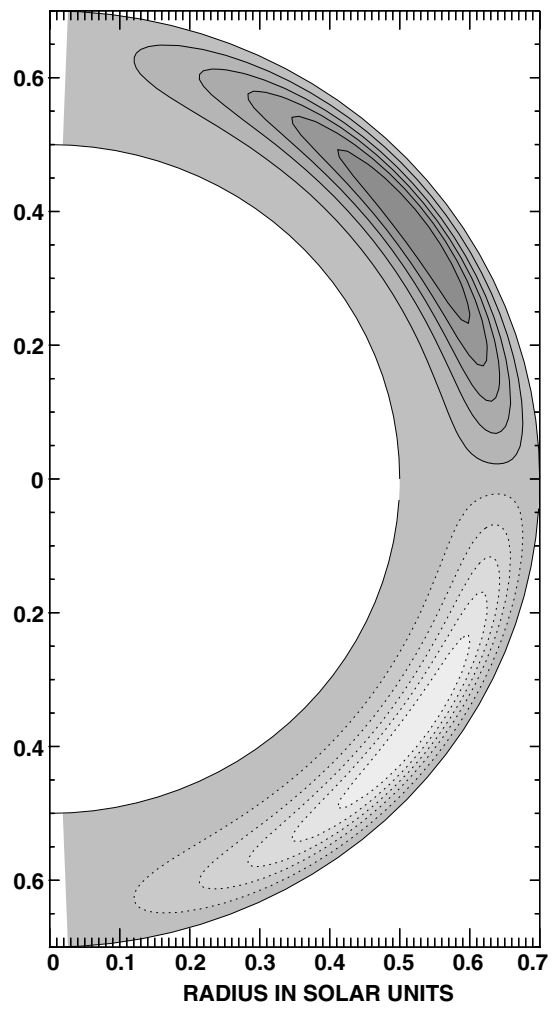

Fig. 1. Contour lines of the toroidal magnetic field strength of the imposed axisymmetric magnetic field if $q=2$ with a positive $B_{\phi}$ in the northern hemisphere and a negative one in the southern hemisphere.

which the high turbulent viscosity and diffusivity turn into nearly microscopic values at the bottom of the convection zone. It is not important for our findings to know at which radius this happens precisely in reality. The inner radius is not meant to have a physical meaning. It is chosen far enough from the radii at which we will place the interesting features of the background state, such as magnetic fields and differential rotation, that it is unimportant for the results. Since the linearized equations decouple for azimuthal Fourier modes $m$, we solve the system with prescribed $m$ and get instability limits for each $m$-mode individually.

The normalization of the magnetic field leads to another parameter, the Lundquist number

$S=\frac{R B_{0}}{\sqrt{\mu \rho} \eta}$

where $R$ is again the solar radius, $B_{0}$ the maximum toroidal magnetic field, $\rho$ and $\eta$ are the density and the magnetic diffusivity in the tachocline in cgs units.

The background magnetic field is purely toroidal and is distributed by

$B_{\phi}=\sin ^{2} \theta \cos \theta \sin ^{2}\left[\pi\left(\frac{r-r_{\mathrm{i}}}{r_{\mathrm{o}}-r_{\mathrm{i}}}\right)^{q}\right]$,

where $q$ controls the radial thickness of the toroidal field belt. We set $q=2$ in most cases, giving a thickness of about 0.1 solar radii. Figure 1 illustrates the configuration. The maximum $B_{\phi}$ sits at a radius of $r=0.641$ and a colatitude of $\theta=55^{\circ}$ in the northern hemisphere. If $q=4$, the maximum is at $r=0.668$. The full radial widths at half-maximum of these profiles are $d=$ $0.073, d=0.044$, and $d=0.025$ for $q=2,4$, and 8, respectively.

The solar magnetic Reynolds number in the tachocline is - in terms of the definition of (3) - about $10^{12}$. We try to achieve time 


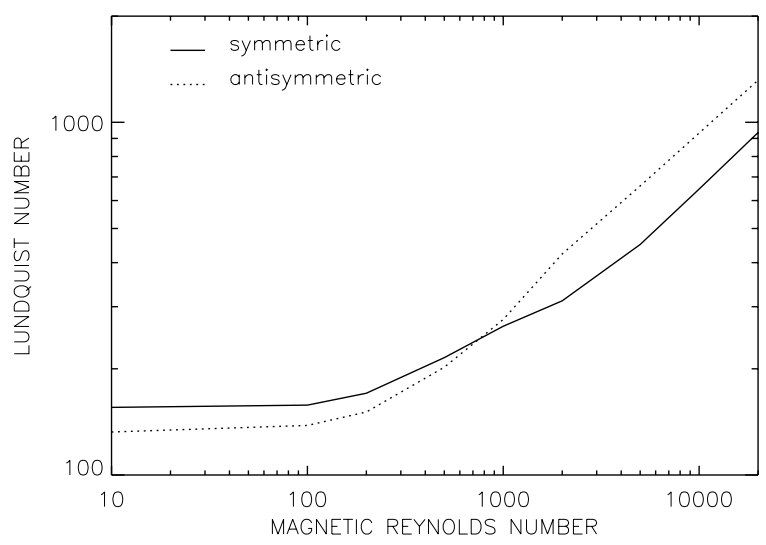

Fig. 2. Lines of marginal stability in a rigidly rotating sphere for different symmetries of the $m=1$ mode with respect to the equator. The solid line is for the solution with a symmetric velocity field; the dotted line is for the antisymmetric velocity field. The magnetic Prandtl number was unity.

series for numerically demanding $\mathrm{Rm}=2 \times 10^{4}$. Magnetic-field belts with $q=2$ can be treated with relatively low resolution, such as 20 Chebyshev and 40 Legendre modes for the decomposition of the meridional structure. Thinner field belts obtained with $q=4$ require significantly higher resolution. A decomposition into 40 Chebyshev and 80 Legendre modes was necessary to achieve numerical stability.

The boundary conditions for the magnetic field $\boldsymbol{b}$ are vacuum conditions for both radial boundaries. The turbulent convection zone on top may easily provide vacuum-like conditions. The inner boundary is actually conducting; however, the conductivity is nearly constant across it. In the context of a finite conductivity in the computational domain, this is far from ideally conducting. In order to prevent artificially strong magnetic fields near the boundary, we also chose vacuum conditions for the inner boundary. The boundary conditions for the $m \geq 1$ flow are always stress-free, whereas an axisymmetric background velocity is prescribed in the models. The velocity fluctuations $\boldsymbol{u}$ do not, of course, change the average rotation velocity of the surfaces of the spherical shell. The boundary conditions also mean that there will be no boundary layers of strong shear near the inner or outer surfaces.

\section{Rigid rotation}

In order to understand the influence of toroidal magnetic fields and differential rotation separately, we first start with a rigidly rotating spherical shell, i.e. $\Omega(r, \theta)=1$.

Symmetric and antisymmetric solutions with respect to the equator are also decoupled in the linearized system investigated here. At a given Rm, we search for the critical Lundquist number at which the growth rate of the perturbation excited is zero. The critical Lundquist numbers for an $m=1$ mode in the range of $\mathrm{Rm}=10$ to 20000 is shown in Fig. 2. The magnetic Prandtl number was $\mathrm{Pm}=1$ in these runs. There is also a magnetic instability without rotation. It occurs at $S=155$ for the symmetric $m=1$ mode and at $S=132$ for the antisymmetric mode. For high magnetic Reynolds numbers, it appears that the symmetric mode dominates. We thus focus on the stability limits of the symmetric mode below, as it is likely to be the interesting one for solar tachocline applications.

The magnetic Prandtl number is much lower than unity in the solar tachocline. According to Stix \& Skaley (1990), we will

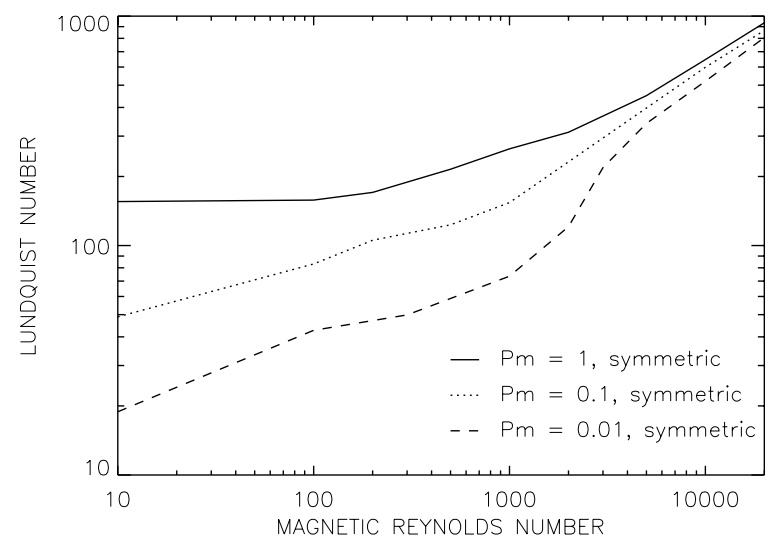

Fig. 3. Lines of marginal stability in a rigidly rotating sphere for various values of the magnetic Prandtl number Pm.

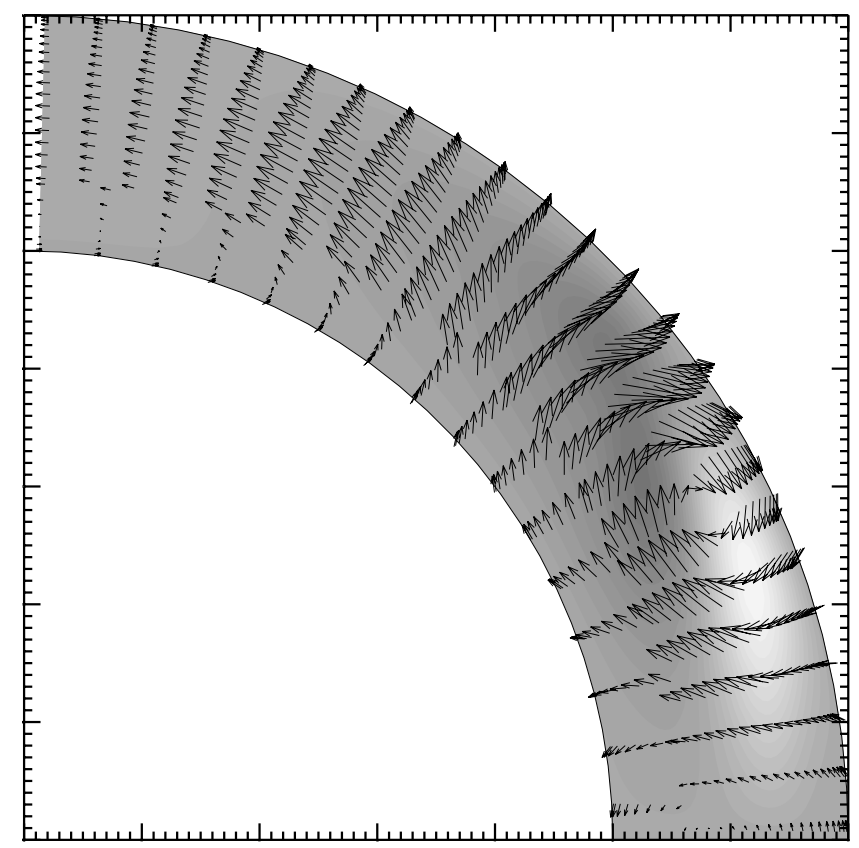

Fig. 4. Magnetic-field perturbation growing in a rigidly rotating sphere at a magnetic Reynolds number of $\mathrm{Rm}=10000$ and a (supercritical) Lundquist number of $S=2000$. The grey scale represents $b_{\phi}$ (without the background profile $B_{\phi}$ of (5), and the arrows are the field vectors projected on the meridional section.

have to reach magnetic Prandtl numbers as low as $\mathrm{Pm}=0.01$ or even slightly smaller. The stability analysis was repeated for $\mathrm{Pm}=0.1$ and $\mathrm{Pm}=0.01$ assuming that the symmetric mode is the most interesting one for solar applications. The lines of marginal stability are shown in Fig. 3. With an antisymmetric background magnetic field as described by (5), we obtain an antisymmetric $m=1$ mode for the magnetic field $\boldsymbol{b}$, too. A vertical cross-section of a growing mode is shown in Fig. 4. Note that the picture changes if one goes to other longitudes of the computational domain.

We cannot access solar magnetic Reynolds numbers in the numerical model. However, the dependence of the critical Lundquist number on $\mathrm{Rm}$ may give us a scaling relation for the extrapolation to a solar magnetic Reynolds number of say $\mathrm{Rm}=10^{12}$. The stability lines in Fig. 3 indicate a convergence to a common slope for all three Pm. This common slope occurs for magnetic Reynolds numbers of 5000-20000. Higher Reynolds 


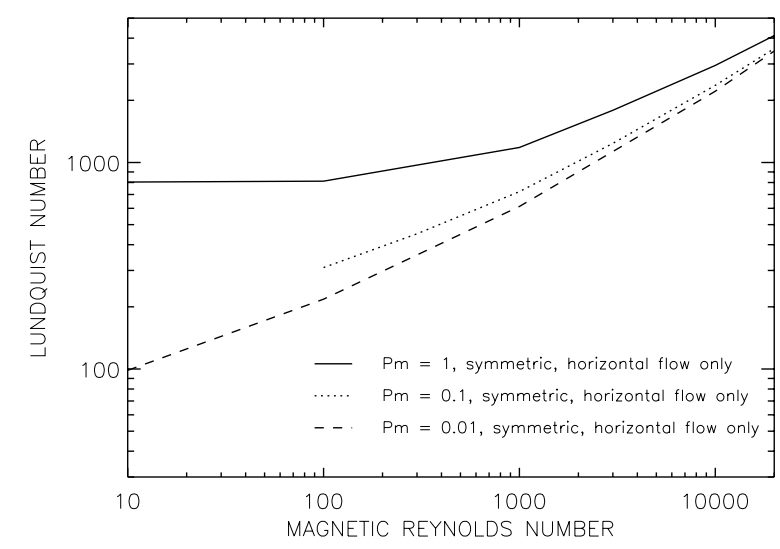

Fig. 5. Lines of marginal stability in a rigidly rotating sphere as in Fig. 3, but with the flow restricted to toroidal motions.

numbers have not yet been accessible by the numerics so far. If we assume that the slopes remain constant for higher Rm, we may express the scaling by

$S_{\text {crit }}=\hat{S} \mathrm{Rm}^{\zeta}$,

where $\zeta$ is the slope in a $\log$ - $\log$ plot and $\hat{S}$ the amplitude of the fit curve. In the particular case shown in Fig. 3, a scaling of

$S_{\text {crit }}=1.6 \mathrm{Rm}^{0.63}$

is found for the high-Rm part of the Pm $=0.01$ line. Because both $S$ and Rm depend on the magnetic diffusivity $\eta$, it is helpful to replace them by their definitions (3) and (4), leading to

$$
\begin{aligned}
B_{\text {crit }} & =\frac{\hat{S} \sqrt{\mu \rho}}{R}\left(R^{2} \Omega_{\mathrm{eq}}\right)^{\zeta} \eta^{(1-\zeta)} \\
& =Q(\zeta) \eta^{(1-\zeta)} .
\end{aligned}
$$

When scaled to a solar tachocline diffusivity of $\eta=3000 \mathrm{~cm}^{2} / \mathrm{s}$ (Stix \& Skaley 1990), we obtained a critical strength of the toroidal magnetic field of $B_{\text {crit }}=12$ Gauss. The density was taken to be $\rho=0.25 \mathrm{~g} / \mathrm{cm}^{3}$ for the tachocline (Guenther et al. 1992). It must be noted how sensitively $Q$ depends on $\zeta$, which is derived from the curves in Fig. 3 at high magnetic Reynolds numbers. There is a factor of 10 change, if $\zeta$ is changed by 0.05 . This is in fact roughly the uncertainty in the determination of $\zeta$.

Since the tachocline is stably stratified to a large extent, the computations were repeated without the development of a poloidal flow. Preventing radial motions entirely corresponds to the case of extremely stable stratifications. Since the numerical scheme solves the toroidal and poloidal potentials of the flow and the field separately, it is as easy to drop one of the equations to imitate the stable stratification.

The stability limits resulting from these calculations with purely horizontal flows are shown in Fig. 5, where the $\zeta$ derived from the slopes of the curves for $\mathrm{Pm}=0.1$ and 0.01 are close to the above value for the full flow. The horizontal flow yields $\zeta=0.59$, but a somewhat higher $\hat{S}$. Equation (8) gives the critical strength of the toroidal magnetic field of $20 \mathrm{G}$, again assuming a diffusivity of $\eta=3000 \mathrm{~cm}^{2} / \mathrm{s}$. This value is practically the same as for the full flow, keeping in mind the general uncertainty of the extrapolation.

\section{Thickness of field belts}

The exponent $q=2$ in definition (5) of the magnetic-field belts yields a radial thickness for the magnetic structure of roughly

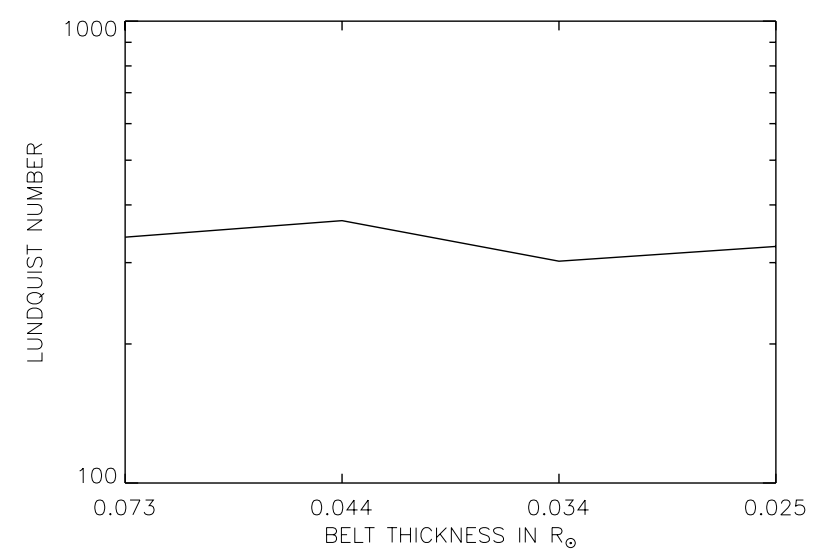

Fig. 6. Critical magnetic-field strengths as a function of the thickness of the field belts. The values were derived for the symmetric $m=1$ mode at $\mathrm{Rm}=5000$ and $\mathrm{Pm}=1$.

$7 \%$ of the solar radius. Higher $q$ produce slimmer belts, and the change in stability can be investigated. In order to achieve numerical stability for very thin belts, we chose a moderate magnetic Reynolds number of $\mathrm{Rm}=5000$ for this test. The full meridional flow was permitted in the calculations.

The dependence of the critical magnetic field in terms of Lundquist numbers on the thickness of the $B_{\phi}$-belts is plotted in Fig. 6. There is no clear trend toward lower or higher stability limits when going to very thin belt thicknesses. The computations may show whether there is a smooth transition to the results of 2D computations on spherical surfaces or to flux-tube simulations. These led to maximum toroidal field strengths of $10^{4}-10^{5}$ Gauss. We assumed that calculations with no radial gradients and/or no radial extent may not be able to fully account for the instability of strong magnetic toroidal fields.

\section{Differential rotation}

\subsection{Latitudinal differential rotation}

The next step consists of adding a latitudinal differential rotation to the spherical shell. The angular velocity $\Omega$ should only depend on the latitude for the moment

$\Omega=\Omega_{\mathrm{eq}}\left(1-\alpha \cos ^{2} \theta\right)$

where $\theta$ is again the colatitude and $\alpha$ a parameter describing the strength of the differential rotation. At $\alpha=1$, the pole is at rest.

We know from hydrodynamic computations that a purely latitudinal differential rotation is unstable for $\alpha>0.28$ (Watson 1981). Now the toroidal field belts of Eq. (5) are added to the differential rotation. The resulting areas of stability are shown in Fig. 7 for two magnetic Reynolds numbers, Rm = 1000 and 10000. The horizontal axis is the ratio of the Lundquist number to the magnetic Reynolds number. This is a useful choice as it does not contain the only unknown in our normalization, the magnetic diffusivity. The ratio is in fact a measure for the ratio of the Alfvén velocity to the rotational velocity at the equator.

At the lower Reynolds number of the two, viscosity is still large enough to increase the limit for hydrodynamic instability (intersection with vertical axis) to about $55 \%$ differential rotation. The area between the two solid lines is the stable region for the $\mathrm{Rm}=1000$ case.

At $\mathrm{Rm}=1000$, the latitudinal differential rotation is stabilized by a relatively weak magnetic field. There is actually a certain magnetic field strength for which even $100 \%$ differential 


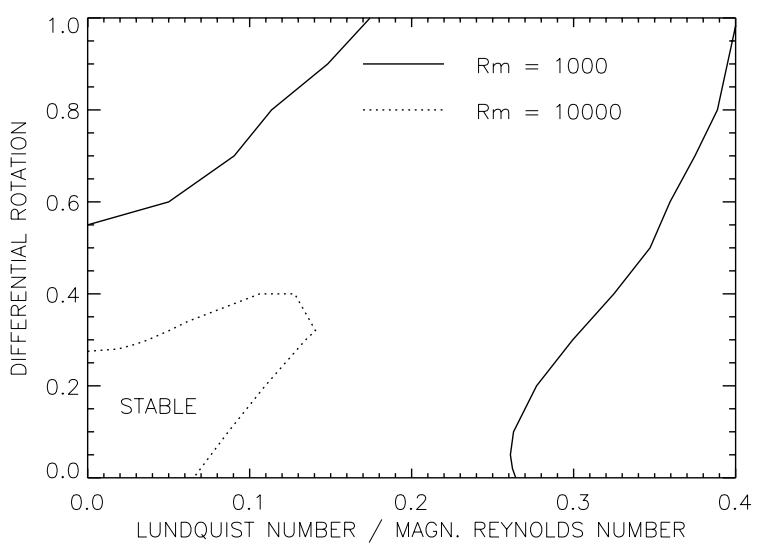

Fig. 7. Lines of marginal stability for toroidal magnetic field belts in a differentially rotating spherical shell with $\Omega \sim\left(1-\alpha \cos ^{2} \theta\right)$. The value of $\alpha$ is given on the vertical axis. The magnetic Reynolds number of these computations was fixed at $\mathrm{Rm}=1000$ for the solid line and at $\mathrm{Rm}=10000$ for the dotted line.

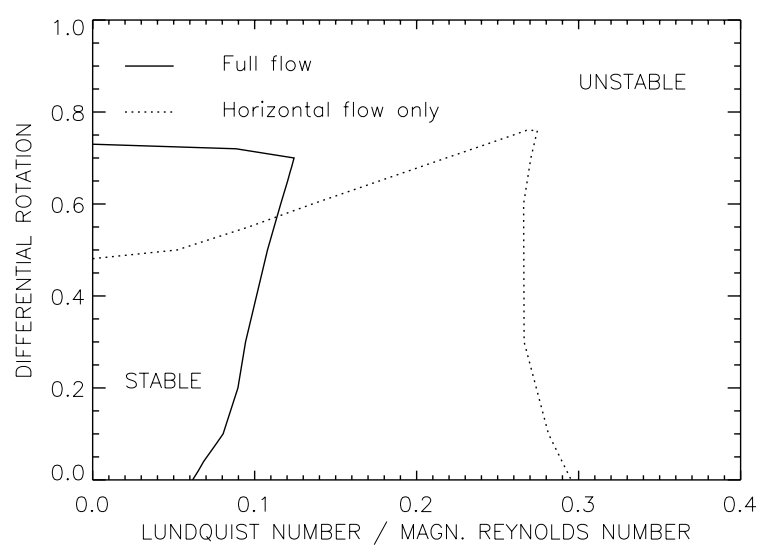

Fig. 8. Lines of marginal stability for toroidal magnetic field belts $(q=2)$ in a differentially rotating spherical shell at a magnetic Reynolds number of $\mathrm{Rm}=10000$. The values at the vertical axis are $\alpha$ from (10). The dashed line shows the marginal stability of a horizontal flow, whereas the solid line is the stability limit for full toroidal and poloidal flow.

rotation (resting pole) is stable. However, very strong magnetic fields destabilize the rotation profile as a consequence of what we recognise as Tayler instability. Starting from the purely magnetic instability on the horizontal axis of the graph, it is found that differential rotation stabilizes the Tayler branch. There is no instability at all for the ratios $S / \mathrm{Rm}$ of 1.8 to 2.6.

At $\mathrm{Rm}=10000$, the totally stable region disappears. There is no stability at ratios of Lundquist number to magnetic Reynolds number higher than 0.14 . However, the stabilizing effect of a weak magnetic field on the hydrodynamic instability, as well as the stabilizing effect of a weak differential rotation on the magnetic instability, is still evident.

\subsection{Full differential rotation}

We now consider a differential rotation that depends on both latitude and radius. The hydrodynamic instability of such a rotation profile emerges at stronger differential rotation than for purely latitudinal differential rotation Arlt et al. (2005).

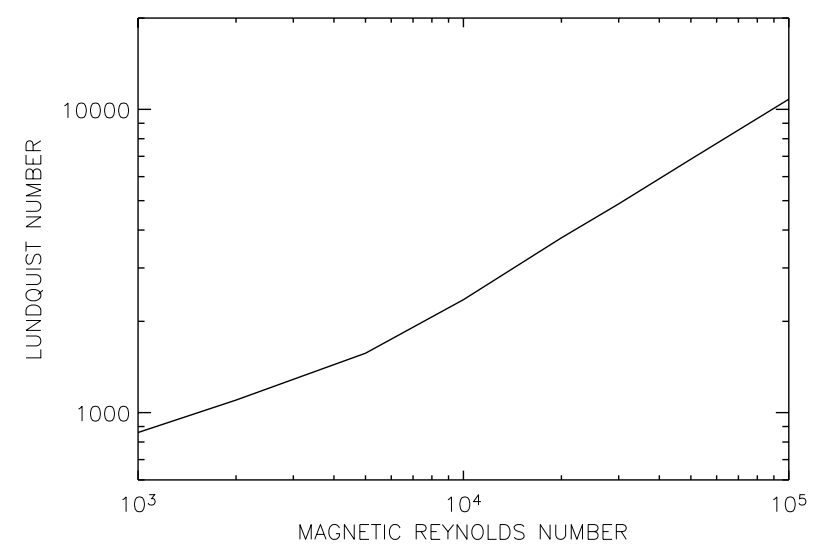

Fig. 9. The same graph as in Fig. 3 but for the full differential rotation profile $\Omega(r, \theta)$ and $\mathrm{Pm}=1$. The slope of this full problem is now $\zeta=$ 0.66 .

The full differential rotation profile is now

$\Omega= \begin{cases}\Omega_{\mathrm{eq}}\left[1-\alpha \cos ^{2} \theta-\alpha\left(\frac{1}{4}-\cos ^{2} \theta\right) \frac{r_{\mathrm{o}}-r}{r_{\mathrm{o}}-r_{\mathrm{t}}}\right] & \text { if } r>r_{\mathrm{t}} \\ \Omega_{\mathrm{eq}}\left[1-\frac{\alpha}{4}\right] & \text { if } r \leq r_{\mathrm{t}}\end{cases}$

where $r_{\mathrm{t}}$ is the radius of the bottom of the tachocline below which no differential rotation is prescribed. This is different from the configuration of Arlt et al. (2005) in that there is an extended radial zone now in which the angular velocity is constant. The value of the tachocline radius was set to $r_{\mathrm{t}}=0.6$ for the relatively thick magnetic-field belts constructed by $q=2$ in (5), and was set to $r_{\mathrm{t}}=0.65$ for the $q=4$ case described at the end of this section.

It is now essential to check whether the additional differential rotation changes the slope $\zeta$ as compared with the the rigidly rotating sphere. Figure 9 shows the line of marginal stability for the full differential rotation with $\alpha=0.2$ and $\mathrm{Pm}=1$. We find $\zeta=0.66$, which together with a new $\hat{S}$, delivers a maximum toroidal magnetic field strength of 100 Gauss. This is our main result for the stability of toroidal fields combined with a tachocline-type rotation.

The stability diagram in Fig. 8 showing the amplitude of differential rotation versus magnetic field strength illustrates the two regimes of the hydrodynamic and magnetic instabilities. We have plotted the lines of marginal stability for a magnetic Reynolds number of $\mathrm{Rm}=10000$ in terms of the surface differential rotation parameter $\alpha$ versus the ratio of Lundquist number to Reynolds number (with the latter being fixed). The solid line gives the marginal stability for computations with a full flow, as it would be expected in an unstratified sphere. The dashed line is for a flow restricted to horizontal motions or, in technical terms, without poloidal flow.

Figure 8 shows that there is a clear separation of the two instabilities. If the magnetic field is small, the hydrodynamic instability (Watson instability) is nearly unaffected, as indicated by the horizontal branch of the stability line. The field strength necessary for the magnetic instability (Tayler instability) alone is found on the horizontal axis of the diagram. This minimum magnetic field does not change significantly if differential rotation is added. This is the vertical branch of the stability line.

In the case of a purely horizontal flow, we observe a stabilizing effect by weak magnetic fields, which slightly suppresses the Watson instability at not too large magnetic field strengths. This effect, however, becomes smaller when one goes to larger 


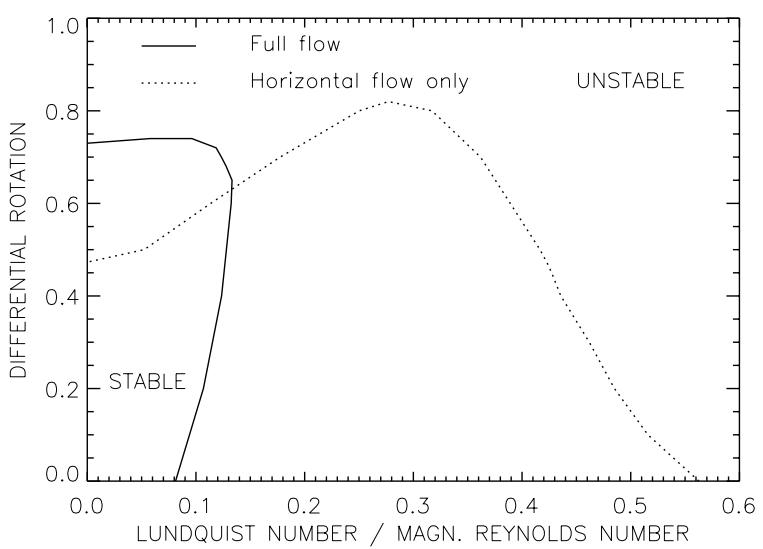

Fig. 10. Lines of marginal stability for thin toroidal magnetic field belts $(q=4)$ in a differentially rotating spherical shell at a magnetic Reynolds number of $\mathrm{Rm}=10000$. As in Fig. 8, the dashed line is for a horizontal flow, whereas the solid line is for the full toroidal and poloidal flows.

magnetic Reynolds numbers, that is smaller magnetic diffusivities. The marginal line with a slightly positive slope merges with the line of marginal stability coming from the purely magnetic part of the diagram at $\alpha=0$. There is no stability at all for $S / \mathrm{Rm}>0.30$.

The thickness of the magnetic-field belts has been roughly $7 \%$ of the solar diameter in most of the results shown up to this point in the paper, except in Sect. 4. The same graph as in Fig. 8 has been repeated with $q=4$ in Eq. (5). This exponent results in a thickness of about 0.04 solar radii. The lines of marginal stability are shown in Fig. 10. The left part of the diagram changes very little. The full-flow stability line is nearly identical to the one for $q=2$. Also the slight stabilization of differential rotation with magnetic fields occurs again in the analysis of purely horizontal flows. The strongest magnetic field is larger, however, and may reach $S / \mathrm{Rm}=0.56$ for rigid rotation $(\alpha=0)$.

\section{Nonlinear evolution}

While this paper concentrates on the linear stability of toroidal magnetic fields in the tachocline, we also provide a short numerical experiment with the nonlinear evolution of the instability in this section. The full, diffusive induction equation is employed, together with the incompressible, viscous momentum equation. Like in the linear models, there is no buoyancy. The axisymmetric background differential rotation is kept constant, while all other quantities are evolved in time. The $m=0$ of the azimuthal velocity is therefore kept constant.

A total of 30 Fourier modes were used to construct the azimuthal structures of flow and magntic field. Figure 11 shows the first 9 modes of the toroidal field, including the axisymmetric one. Note that the axisymmetric part of the toroidal field does change, whereas the axisymmetric part of the azimuthal flow is the only quantity that is kept constant.

As the instability sets in, a growth in higher $m$-modes is observed. The nonlinear coupling between the modes is effective in producing rapid growth of small-scale structures. The nonlinear evolution indicates that the system develops into a turbulent state in which turbulent diffusion will destroy the initially large-scale field.

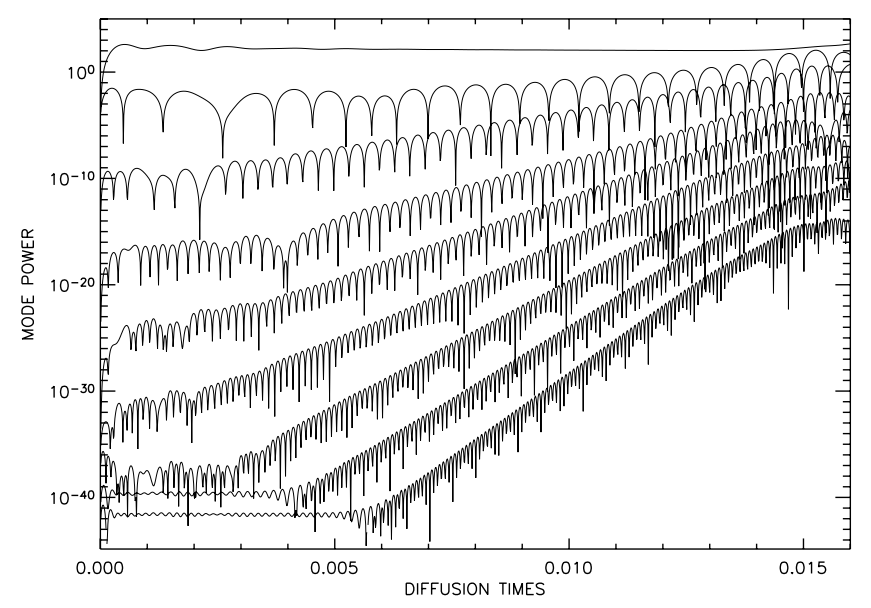

Fig. 11. Nonlinear evolution of various azimuthal modes with time. The individual lines refer to the modes $m=0$ to $m=8$, from top to bottom. The background $\Omega$-profile is kept constant in this computation.

\section{Discussion}

We studied the stability of toroidal magnetic fields in spherical geometry. Three steps were taken to understand this stability. Belts of toroidal magnetic fields were first placed in a rigidly rotating spherical shell. A latitudinal differential rotation was then added to the configuration, and in the final step, a solar-like differential rotation with $\Omega$ depending on $r$ and $\theta$ was combined with the toroidal field belts.

A good estimate for the maximum possible toroidal magnetic field is already given by the calculations of a rigidly rotating sphere. Higher magnetic Reynolds numbers are accessible in that simpler setup. The dependence of critical field strength on Reynolds number appears to be a power law. The extrapolation to the microscopic solar diffusivity in the tachocline leads to maximum stable toroidal magnetic fields of the order of 10 Gauss. The full differential rotation $\Omega(r, \theta)$ in the tachocline leads to an upper limit of 100 Gauss.

Hydodynamic stability of the tachocline in a 3D setup was found by Arlt et al. (2005). Starting from that point with $S=0$, we find that the hydrodynamic limit is weakly influenced by additional, small toroidal magnetic fields. If the fields are strong enough to excite a magnetic instability, this in turn is weakly influenced by the amplitude of the differential rotation. In other words, the addition of differential rotation to the magnetic instability does not change the critical field strengths dramatically. This is best demonstrated in Figs. 8 and 10. The interaction of the hydrodynamic and the magnetic regimes of the instability may provide nonlinear phenomena that will be an interesting subject for future investigations.

The investigation of the Lithium depletion problem showed that the microscopic magnetic diffusivity may not be sufficient for explaining the observed values. A slightly turbulent state with a viscosity 10 times above the microscopic value is more likely (Vauclair et al. 1978; Lebreton \& Maeder 1987). If we also apply this factor to the magnetic diffusivity, it increases the maximum stable field strength to a slight degree. The change in critical magnetic field strengths is given by $\eta^{0.4}$ and is thus not more than one order of magnitude due to the suggested weak tachocline turbulence.

Maximum stable magnetic fields of roughly 100 Gauss are found with little dependence on the actual rotation profile used. Even though the precise limit for the toroidal magnetic field may be different by one order of magnitude from the one derived here, 
stable toroidal fields of $10^{5} \mathrm{G}$ appear very unlikely. The fact that the tachocline is stably stratified does not add considerably to the stability of the magnetic fields. A decrease in the radial thickness of the magnetic-field belts does not lead to increasing critical magnetic fields. Non-3D computations may thus give inadequate results for the stability of magnetic fields in the solar tachocline.

The instability of $10^{5} \mathrm{G}$ toroidal fields will have consequences on the dynamo process causing the 22-yr cycle of the solar magnetic field. The tachocline will then not be a location where very strong magnetic fields can be accumulated and stored. The possibility of a dynamo distributed over the convection zone, or even restricted to its upper part, was recently revived by Brandenburg (2005). This idea gains support from our finding that only moderate magnetic fields are stable in the solar tachocline. A dynamo much closer to the surface also generates toroidal magnetic fields, and we can estimate the stability of such large-scale fields using turbulent values for the magnetic Reynolds number and the magnetic Prandtl number. The former, $\mathrm{Rm}_{\mathrm{t}}$, is about 1000-2000, whereas $\mathrm{Pm}_{\mathrm{t}}$ is unity or a bit less than that (Yousef et al. 2003). The maximum stable Lundquist number is $200-300$ as read from Fig. 3 and from the $30 \%$ differential rotation in Fig. 7. With a density of about $0.01 \mathrm{~g} / \mathrm{cm}^{3}$ and a turbulent diffusivity of $\eta_{\mathrm{t}} \sim 10^{12} \mathrm{~cm}^{2} / \mathrm{s}$, these values convert to 1000-1500 Gauss, which is enough to permit the toroidal fields of the suggested surface dynamo (large-scale field of a few hundred Gauss).

The idea of a tachocline formation from an internal fossil magnetic field is not affected by the instability studied here. The effect of an internal field was calculated by Rüdiger \& Kitchatinov (1997) and Gough \& McIntyre (1998). The former found a toroidal magnetic field balancing with the differential rotation in the tachocline at about 200 Gauss. Keeping a weak turbulent diffusion in the tachocline in mind, this field strength appears roughly compatible with what we found to be the maximum stable toroidal fields in the tachocline. The necessary poloidal fields in Gough \& McIntyre (1998) were somewhat higher, as they asked for a tachocline thickness of $0.018 R$. Once we assume a $0.04 R$ tachocline, their results are very similar to those of Rüdiger \& Kitchatinov (1997) and thus not likely to be subject to the toroidal-field instability discussed here.

Acknowledgements. A.S. thanks the Deutsche Forschungsgemeinschaft for the support by grant No. Ru 488/15-1.

\section{References}

Arlt, R., Sule, A., \& Rüdiger, G. 2005, A\&A, 441, 1171

Brandenburg, A. 2005, ApJ, 625, 539

Choudhuri, A. R., \& Gilman, P. A. 1987, ApJ, 316, 788

D'Silva, S., \& Choudhuri, A. R. 1993, A\&A, 272, 621

Dikpati, M., Cally, P. S., \& Gilman, P. A. 2004, ApJ, 610, 597

Gilman, P. A., \& Fox, P. A. 1997, ApJ, 484, 439

Gough, D., \& McIntyre, M. E. 1998, Nature, 394, 755

Ferriz-Mas, A., \& Schüssler, M. 1995, Geophys. Astrophys. Fluid Dyn., 81, 233

Guenther, D. B., Demarque, P., Kim, Y.-C., \& Pinsonneault, M. H. 1992, ApJ, 387,372

Hollerbach, R. 2000, Int. J. Num. Meth. Fluids, 32, 773

Lebreton, Y., \& Maeder, A. 1987, A\&A, 175, 99

Rüdiger, G., \& Kitchatinov, L. L. 1997, Astron. Nachr., 318, 273

Rüdiger, G., Hollerbach, R., Stefani, et al. 2006, ApJ, 649, 145

Stix, M., \& Skaley, D. 1990, A\&A, 232, 234

Tayler, R. J. 1973, MNRAS, 161, 365

Vauclair, S., Vauclair, G., Schatzman, E., \& Michaud, G. 1978, ApJ, 223, 567

Watson, M. 1981, GAFD, 16, 285

Yousef, T. A., Brandenburg, A., \& Rüdiger, G. 2003, A\&A, 411, 321 